\title{
Two-stage exchange for PJI with co-existing cerclages for fracture: higher rates of early re-infections and difficult to treat microbes
}

\author{
Daniel Karczewski ${ }^{1}$ D $\cdot$ Maximilian Müllner ${ }^{1} \cdot$ Christian Hipfl $^{1} \cdot$ Carsten Perka $^{1} \cdot$ Michael Müller $^{1}$
}

Received: 1 February 2021 / Accepted: 13 November 2021 / Published online: 28 January 2022

(c) The Author(s) 2022

\begin{abstract}
Introduction Periprosthetic joint infections (PJI) with osteosynthesis material for contemporaneous fractures are a challenging, yet poorly described condition. This study will analyze PJI with co-existing fractures treated with cerclages and two-stage exchange.

Materials and methods Patients with and without cerclages for coexisting periprosthetic fractures, undergoing two-stage exchange for PJI of hip or knee, between 06/2013 and 02/2016, were compared concerning baseline characteristics and re-infection rate in the course of a 2 year follow-up. All patients were treated with a standardized two-stage protocol. A PJI was defined according to the EBJIS criteria. All foreign material, including cerclages, was sent in for sonication for microbiological analysis.

Results Ninety-six patients treated with two-stage exchange for PJI could be included. Co-existing fractures treated with cerclage were identified in nine patients (9.3\%, study group). Diaphyseal femoral simple in five cases (AO2A3) and proximal intertrochanteric in three cases (AO1A3) were the leading fracture locations. In one patient, cerclage implantation was performed prior to prosthesis explantation, in six, during prosthesis explantation, and in two, in the course of prosthesis reimplantation. The study group showed a significantly higher rate of difficult to treat microbes $(44.4 \% ; 8.0 \% ; p=.001)$, Charlson Comorbidity Index $(5.4 ; 3.7 ; p=.033)$, relapse infections with the same microbe $(22.2 \% ; 1.1 \% ; p=.001)$, and early-onset infections ( $<30$ days) $(11.1 \% ; 1.1 \% ; p=.046)$, than the comparison two-stage exchange group without fractures. In contrast, age (72.5 study group; 68.2 comparison group; $p=.224)$, rate of revisions for PJI in the past (55.5\%; 51.7\%; $p=.827)$, and total re-infection rate $(22.2 \% ; 10.3 \% ; p=.287)$ did not show a difference.

Conclusion PJI with co-existing cerclages for fractures were associated with multi-resistant microbes, relapse by the same microbe and early-onset re-infections. Cerclages might be considered a potential source of re-infection during a two-stage exchange. However, statistical weaknesses and a small study group must be considered limitations of the study.
\end{abstract}

Keywords Periprosthetic joint infection $\cdot$ Fracture $\cdot$ Cerclage $\cdot$ Two-stage exchange

\section{Introduction}

A periprosthetic joint infection (PJI) is one of the most devastating complications of arthroplasty. Due to an aging society and an increasing acceptance for arthroplasties in younger patients, both the absolute number of arthroplasties and number of PJI has increased in recent years $[1,2]$. PJI with contemporaneous fractures was described as "the

Daniel Karczewski

daniel.karczewski@charite.de

1 Center for Musculoskeletal Surgery, Department of Orthopaedic Surgery, Charité- University Medicine, Charitéplatz 1, 10117 Berlin, Germany worst-case scenario" by Müller et al. in a case series of eight patients, given the necessity to eradicate an infection on the one hand, and to stabilize a fracture using osteosynthesis material, with subsequent increased infection risk, on the other hand [3]. Existing clinical studies were able to show that arthroplasties implanted due to a fracture, for example, following a femoral neck fracture, have a higher risk of developing a PJI compared to the ones implanted because of a degenerative joint disorder $[4,5]$. Besides, fracturesamong other causes-are a known general risk factor for development of a new infection after joint arthroplasty [6, 7]. However, up to this point, there are limited studies available concerning contemporaneous PJI and fractures. 
This study analyzes a two-stage exchange protocol for patients with a PJI and a contemporaneously co-existing fracture treated with cerclages. As opposed to prior case series and reports, this study will compare outcome and characteristics of the patient cohort with patients treated with the same two-stage protocol and in the same department, but without co-existing fractures and cerclage treatment.

\section{Materials and methods}

\section{Study design}

The data were collected retrospectively out of a digital data bank, while all patients included in the study were followed up prospectively using a standardized protocol. The study was approved by the university's ethics committee, including human and animal rights.

\section{Inclusion criteria}

Criteria for inclusion were: (1) confirmed diagnosis of PJI treated with a two-stage exchange protocol, (2) a contemporaneous periprosthetic fracture treated with cerclage, (3) involvement of hip or knee joint, (4) and treatment from 06/2013 to 02/2016 in our department. Patients with (1) different PJI treatment strategies (one-stage exchange, debridement and implant retention), (2) fracture treatments other than cerclage (plates, screws), and (3) cerclages for extended trochanteric osteotomy (ETO) were excluded.

\section{Follow-up}

Our departments follow-up schedule is based on followups every 12 weeks within the first year, and then every 6 months. Analyzed parameters at the time of surgery included fracture classification according to AO [8], microbe spectrum, patient age, age-adjusted CCI (Charlson Comorbidity Index) [9], and prior revisions. Follow-up parameters included re-infection and total re-revision rate during a 2-year follow-up.

\section{Treatment protocol}

Every patient was treated with a long interval two-stage exchange for PJI [10]. The standardized protocol included a spacer less Girdlestone resection arthroplasty for all patients, followed by 2 weeks of i.v. antibiotics (AB). After 4 further weeks of oral $\mathrm{AB}$, and if no signs of local or systemic infection were present, the prosthesis was reimplanted. Following the reimplantation, i.v. biofilm-active $\mathrm{AB}$ were administered for 1 week, and the protocol continued with 5 weeks of oral biofilm-active antibiotics, until a total of 12 weeks of continuously $\mathrm{AB}$ was completed. Initial calculated $\mathrm{AB}$ consisted of ampicillin/sulbactam $(3 \times 3 \mathrm{~g}$ i.v. $)$ and additional vancomycin $(2 \times 1 \mathrm{~g}$ i.v. $)$ in septic cases, MRSA-carriers, multiple prior surgeries, and suspected low-grade infection. As soon as a microbe was detected, targeted therapy was started, as proposed by Izakovicova and Trampuz et al. [11].

Germ detection was based on a standardized diagnostical algorithm. During the operation, at least four tissue samples were obtained. In addition, all foreign materials were sent in for sonication. The microbiological results summarized represent microbes identified through the total of all diagnostical tools.

\section{Definitions}

A PJI was defined based on the European Bone and Joint Infection Society (EBJIS) [12]: (1) fistula or purulence around prosthesis, (2) leukocyte count in synovial fluid $>2000 / \mu 1$ or $>70 \%$ granulocytes (polymorphonuclear leukocytes), (3) periprosthetic tissue histology Krenn and Morawietz type 2 or type 3 [13], (4) microbial growth in synovial fluid or $\geq$ two tissue samples or sonication fluid. Re-infection was considered an absence of success following the Delphi-consensus criteria [14]: (1) wound healing without fistula, drainage, or pain, (2) no subsequent surgical intervention for infection after reimplantation surgery, (3) no PJI-related death (sepsis, necrotizing fasciitis). In addition, all re-infections were differentiated into new infections with involvement of a new microbe and relapses caused by the priorly identified microbe (Zmistowski and Parvici et al.) [15]. A symptom onset less than 30 days since the last surgery was considered an early infection [11]. Difficult to treat organisms (DTT) were defined as resistant to biofilm-active antimicrobials, including Rifampin-resistant staphylococci, Ciprofloxacin-resistant Gram-negative bacteria, and Candida [16].

\section{Statistics}

Statistical evaluation was performed using SPSS (SPSS Inc., Chicago, IL, USA). Significance was considered $p<0.05$. Significance tests for independent groups were used for calculation, including the $t$ test and Mann-Whitney $U$ test for continuous, and the Chi-square test for categorical variables.

\section{Results}

Nine patients treated with both two-stage exchange for PJI and cerclage for fractures could be included. In two patients, two different fracture localizations were present. Thus, a total of 11 fractures were identified in nine patients. In one patient, cerclage implantation was performed before 
the prosthesis explantation, in six patients cerclages were implanted in the course of the prosthesis explantation, and in two cases, cerclages were implanted in the course of the prosthesis reimplantation. Fractures were all located in the femur (AO 3) with $2 \mathrm{~A} 3$ as leading fracture type in five cases (2 Diaphyseal segment, A Simple, 3 Transverse $<30^{\circ}$ ), followed by $1 \mathrm{~A} 3$ in three cases (1 Proximal end segment, A Trochanteric region, 3 Intertrochanteric), $3 \mathrm{~A} 2$ in two cases (3 Distal end segment, A Extraarticular, 2 Simple), and 2B2 in one case (2 Diaphyseal segment, B Wedge, 2 Intact wedge) (Table 1).

Table 1 Fracture characteristics

\begin{tabular}{ll}
\hline $\begin{array}{l}\text { Patients with cerclage implantation before prosthesis } \\
\text { explanation }\end{array}$ & 1 \\
AO classification of treated fracture & $3 \mathrm{~A} 2$ \\
Average number of cerclages & 1 \\
$\begin{array}{l}\text { Patients with cerclage implantation during prosthesis } \\
\text { explanation }\end{array}$ & 6 \\
AO classification of treated fractures & $2 \mathrm{~A} 3 \times 3$ \\
& $1 \mathrm{~A} 3 \times 3$ \\
& $2 \mathrm{~B} 2$ \\
& $3 \mathrm{~A} 2$ \\
Average number of cerclages & 1.83 \\
Patients with cerclage implantation during reimplantation & 2 \\
AO classification of treated fractures & $2 \mathrm{~A} 3 \times 2$ \\
Average number of cerclages & 2 \\
\hline
\end{tabular}

9.3\% of all two-stage exchanges had a fracture as an additional complication ( 9 of 96 patients) (Table 2). No significant differences between two-stage exchange cases with $(n=9)$ and without $(n=87)$ an additional fracture could be identified concerning age (72.5 years; $68.2 ; p=0.224)$, previous revisions for PJI $(55.5 \% ; 51.7 \% ; p=0.827)$, duration of hospital stay after surgery (33.8 days; 29.6; $p=0.389$ ), total re-infection rate $(22.2 \% ; 10.3 \% ; p=0.287)$, and total re-revision rate $(44.4 \% ; 26.4 \% ; p=0.253)$ in a 2 -year followup. In contrast, two-stage exchanges with additional fractures showed a significantly higher CCI $(5.4 ; 3.7 ; p=0.033)$, rate of early-onset infection $(11.1 \% ; 1.1 \% ; p=0.046)$ and relapse infections with the same microbe $(22.2 \% ; 1.1 \%$; $p=0.001$ ) compared to non-fracture cases. In addition, they showed a trend towards a later reimplantation after explantation (83.4 days; 64.9 days; $p=0.099$ ).

Coagulase-negative Staphylococci and Cutibacterium spp. were the leading microbes identified in both study and comparison group (Table 3). Patients treated with additional cerclages for fracture in the course of the two-stage exchange demonstrated a significantly higher rate of DTT microbes $(44.4 \% ; 8.0 \% ; p=0.001)$ and Gram-negative rods $(p=0.001)$. In both groups, high rates of polymicrobial cases were identified (47.1\% and $66.6 \%)$.

Cerclage material was introduced in the course of the second stage/reimplantation in two cases, thus not allowing for a sonication of cerclage material. In the remaining seven cases, cerclage material was exchanged in five patients (in
Table 2 Two-stage exchange with and without additional fractures

\begin{tabular}{|c|c|c|c|}
\hline Two-stage exchanges & Fracture/cerclage group & Comparison group & $p$ value \\
\hline Patients $(n)$ & 9 & 87 & - \\
\hline Knee/hip, $(n)$ & $3 / 6$ & $44 / 43$ & $.325^{\mathrm{c}}$ \\
\hline Males/females, $(n)$ & $3 / 6$ & $35 / 52$ & $.687^{\mathrm{c}}$ \\
\hline $\operatorname{Age}^{\mathrm{a}}$ & $72.5 \pm 5.0$ & $68.2 \pm 10.3$ & $.224^{\mathrm{d}}$ \\
\hline Age adjusted $\mathrm{CCI}^{1}$ & $5.4 \pm 1.9$ & $3.7 \pm 2.1$ & $.033^{\mathrm{e}}$ \\
\hline Previous septic revision rate, $(n)$ & 5 & 45 & $.827^{\mathrm{c}}$ \\
\hline Duration of hospital stay after surgery (days) ${ }^{a, b}$ & $33.8 \pm 5.9(25-44)$ & $29.6 \pm 14.2(10-115)$ & $.389^{\mathrm{d}}$ \\
\hline Time between ex- and reimplantation (days) ${ }^{\mathrm{a}}$ & $83.4 \pm 50.4(23-174)$ & $64.9 \pm 28.6(7-198)$ & $.099^{\mathrm{d}}$ \\
\hline \multicolumn{4}{|l|}{ 2-year follow-up } \\
\hline Total re-infection rate, $(n)$ & 2 & 9 & $.287^{\mathrm{c}}$ \\
\hline $\begin{array}{l}\text { Early-onset re-infection }(<30 \text { days after final } \\
\text { treatment), }(n)\end{array}$ & 1 & 1 & $.046^{c}$ \\
\hline Relapse, $(n)$ & 2 & 1 & $.001^{c}$ \\
\hline New-infection, $(n)$ & 0 & 8 & $.342^{\mathrm{c}}$ \\
\hline Total re-revision rate, $(n)$ & 4 & 23 & $.253^{\mathrm{c}}$ \\
\hline
\end{tabular}

A $p$ value $<0.05$ was considered significant and printed in bold

${ }^{\mathrm{a}}$ Mean $\pm \mathrm{SD}$ (range)

${ }^{b}$ Duration of stay in days after explanation and reimplantation was added to a common sum

${ }^{\mathrm{c}}$ Chi-square test

${ }^{\mathrm{d}} t$ test

${ }^{\mathrm{e}}$ Mann-Whitney $U$ test 
Table 3 Microbe spectrum

\begin{tabular}{llll}
\hline Two-stage exchanges & $\begin{array}{l}\text { Compari- } \\
\text { son group }\end{array}$ & $\begin{array}{l}\text { Frac- } \\
\text { ture } \\
\text { group }\end{array}$ & $p$ value $^{\mathrm{a}}$ \\
\hline Patients $(n)$ & 87 & 9 & - \\
Coagulase-negative Staphylococci $(n)$ & 47 & 5 & .930 \\
Cutibacterium spp. $(n)$ & 17 & 3 & .332 \\
Staphylococcus aureus $(n)$ & 16 & 2 & .779 \\
Enterococcus spp. $(n)$ & 9 & 2 & .287 \\
Streptococcus spp. $(n)$ & 8 & 1 & .851 \\
Gram-negative rods $(n)$ & 4 & 2 & $\mathbf{. 0 3 8}$ \\
Candida spp. $(n)$ & 1 & 2 & $\mathbf{. 0 0 1}$ \\
Further/unclassified $(n)$ & 15 & 3 & .239 \\
DTT $(n)$ & 7 & 4 & $\mathbf{. 0 0 1}$ \\
Monomicrobial $(n)$ & 41 & 2 & .153 \\
Polymicrobial $(\geq 2$ microbes) $(n)$ & 40 & 6 & .237 \\
Culture negative $(n)$ & 6 & 1 & .643 \\
\hline
\end{tabular}

A $p$ value $<0.05$ was considered significant and printed in bold

${ }^{\mathrm{a}}$ Chi-square test

one case together with the old prosthesis in the first stage, in the remaining cases, cerclages were introduced in the course of the first stage and exchanged or removed in the second procedure), and sent in for sonication. Microbe detection out of the five sonication samples was possible only in the case in which the cerclage was already present prior to the first stage, and also exchanged in the first stage. Two re-infections were noted in the cerclage group. In one case, the cerclage was removed in the course of the prosthesis reimplantation, and thus no cerclage could be sent in for sonication in the course of the re-infection diagnosis. In the second case, cerclage material could be analyzed for microbes via sonication, and identified Staphylococcus aureus as re-infection microbe, thus resembling the originally identified microbe spectrum.

\section{Discussion}

PJI with a co-existing fracture is an important and challenging event. In the present study, nearly one in ten patients treated with a two-stage protocol for PJI had an additional fracture treated with a cerclage at the time of diagnosis or during the treatment itself. Exact data concerning prevalence of PJI and co-existing fractures are unknown, and can only be deducted from aseptic cases, where the rate of fractures after prosthetic hip revision is $4 \%$ [17], the one after knee prosthesis revision up to $30 \%$ [18]. Although exact demographics are undetermined yet, osteoporosis and metastasis in elderly patients on the one, and more active and mobile patients on the other hand, will likely also increase the number of fractures in combination with PJI in the nearby future.

The most important fracture characteristics in our study included a strong preference towards the hip joint and femur, as well as intraoperatively caused fractures. The femur as leading fracture location is typical for periprosthetic fractures in both hip and knee [19]. Besides, several described general risk factors for fractures are present in our patients such as an age older 70 years, prosthesis loosening and a poor overall patient condition [20, 21].

The present study was able to show that a combined therapy using a two-stage exchange and cerclages can efficiently treat PJI with co-existing fractures, resulting in no significantly ( $p=0.287$ ) higher total re-infection rates compared to non-fracture PJI in the course of a 2-year follow-up. However, fracture/cerclage cases demonstrated a significantly higher rate of early-onset infections and relapses by the same microbe. Janz et al. were able to show that cerclages after femoral osteotomy are a risk factor for bacterial colonization during a two-stage septic total hip arthroplasty. This might explain the higher rates of earlyonset and relapse infections found in this study. However, Janz et al.'s study did not include patients with fractures but iatrogenic caused femoral osteotomies (stem mobilization). Besides, the study did not have a standardized comparison group without osteotomies and cerclages [22].

In general, the high rates of Staph. epidermidis (coagulase negative) and Staph. aureus identified in our study are similar to the rates described in most PJI studies without additional fractures [1, 3, 4, 23]. However, compared to prior studies, high rates of DTT such as Candida were identified in the present study. Usually, Candida as a PJI causing microbe is involved in less than $1 \%$ of all PJI [1]. This study is the first one to find a significantly higher DTT rate in two-stage exchanges with fractures treated with cerclage compared to cases without additional fractures $(p=0.001)$. Up to this point, no literature equivalent describing this phenomenon is present. Of the four DTT cases in our study that underwent two-stage revision, one had a preoperative existing fracture, while the others were caused intraoperatively iatrogenic during stem explantation, and in two cases, in the course of the reimplantation. Preoperative existing fractures could facilitate DTT attachment, or DTT cases could react osteolytic/structure damaging, contributing to more intraoperative fractures. In addition, present cerclages might allow for a possible biofilm formation and attachment in the joint. However, the exact relation between DTT and fractures remains undetermined. Age and revision rate for PJI in the past did not seem to influence this correlation. Instead, the small number of patients and a general tendency towards sicker patients (CCI 5.4; 3.7; $p=0.033$ ) should be considered as additional explanations. To allow for an exact statistical 
calculation, a matched pair analysis would be necessary. However, given the overall number of only nine cases in the study group compared to 87 patients in the control group, this analysis itself would be of limited evidence. Further research on a larger patient scale might be necessary in the future.

Despite being the first study to systematically describe characteristics and outcomes of PJI with co-existing factures, structural problems and remaining questions should be critically discussed. A number of questions cannot be answered using the current methods.

As the statistical outcome remains inconclusive, it remains undetermined, if periprosthetic fractures are associated with a poorer outcome. In this context, it also remains unknown if cerclages as biofilm colonized foreign body are the main cause of a persistent infection, or higher rates of relapse infection by the same microbe and fractures are confounding bias of an overall sicker population. Sonication of cerclages might seem like an option to answer this question. However, while sonication of cerclage material might be useful in the course of the first stage (exchange of prosthesis), and thus before the start of antibiosis, the sonication of cerclages removed/ exchanged in the second stage is problematic, as prior antibiosis might cause false-negative results. To give a resume, limitations must be addressed before drawing final conclusions, such as the necessity to exchange cerclage, introduced during prosthesis removal, in the course of the reimplantation of a two-stage exchange.

\section{Conclusion}

Fractures treated with cerclage are an additional complication with a prevalence of about $10 \%$ in two-stage revisions for PJI. The diaphyseal region of the hip was the most prevalent fracture localization, with most fractures being caused intraoperatively iatrogenic. Staph. epidermidis, Cutibacterium and Staphylococcus aureus were the most prevalently identified microbes. Contemporaneously fractures treated with cerclages in the course of a two-stage exchange showed a higher rate of difficult to treat microbes, relapse infections by the same pathogen and early-onset re-infections than patients without additional fractures. Nonetheless, limitations, including limited absolute patient numbers and subsequent statistical weaknesses, must be addressed before drawing final conclusions.
Author contributions DK: concept, primary data, and writing; MM: writing and internal revision; $\mathrm{CH}$ and $\mathrm{CP}$ : administrative support, expert opinion, and writing; MM: concept and writing.

Funding Open Access funding enabled and organized by Projekt DEAL. No specific funding was received.

Availability of data and material Made available upon request.

Code availability Not applicable.

\section{Declarations}

Conflict of interest The authors have no COI to report.

Ethics approval Ethical approval was obtained by the local ethics committee of the university.

Consent to participate Ethics approval was obtained.

Consent for publication Ethics approval was obtained, and all the authors consent.

Open Access This article is licensed under a Creative Commons Attribution 4.0 International License, which permits use, sharing, adaptation, distribution and reproduction in any medium or format, as long as you give appropriate credit to the original author(s) and the source, provide a link to the Creative Commons licence, and indicate if changes were made. The images or other third party material in this article are included in the article's Creative Commons licence, unless indicated otherwise in a credit line to the material. If material is not included in the article's Creative Commons licence and your intended use is not permitted by statutory regulation or exceeds the permitted use, you will need to obtain permission directly from the copyright holder. To view a copy of this licence, visit http://creativecommons.org/licenses/by/4.0/.

\section{References}

1. Tande AJ, Patel R (2014) Prosthetic joint infection. Clin Microbiol Rev 27(2):302-345. https://doi.org/10.1128/CMR.00111-13

2. Kurtz S, Ong K, Lau E, Mowat F, Halpern M (2007) Projections of primary and revision hip and knee arthroplasty in the United States from 2005 to 2030. J Bone Joint Surg. Am vol 89(4):780 785. https://doi.org/10.2106/JBJS.F.00222

3. Müller M, Winkler T, Märdian S, Trampuz A, Renz N, Perka C, Karczewski D (2019) The worst-case scenario: treatment of periprosthetic femoral fracture with coexistent periprosthetic infection-a prospective and consecutive clinical study. Arch Orthop Trauma Surg 139(10):1461-1470. https://doi.org/10.1007/ s00402-019-03263-y

4. Blomfeldt R, Kasina P, Ottosson C, Enocson A, Lapidus LJ (2015) Prosthetic joint infection following hip fracture and degenerative hip disorder: a cohort study of three thousand, eight hundred and seven consecutive hip arthroplasties with a minimum follow-up of five years. Int Orthop 39(11):2091-2096. https://doi.org/10.1007/ s00264-015-2989-y

5. Barbero JM, Montero E, Vallés A, Plasencia MA, Romanyk J, Gómez J (2016) Prosthetic joint infection in patients with hip fracture. Differences from infection of elective prosthesis. Rev Esp Quimioter 29(5):273-277 
6. De Dios M, Cordero-Ampuero J (2015) Risk factors for infection in total knee artrhoplasty, including previously unreported intraoperative fracture and deep venous thrombosis. Rev Esp Cir Ortop Traumatol 59(1):36-43. https://doi.org/10.1016/j.recot.2014.07. 007

7. Fernandes A, Dias M (2013) The microbiological profiles of infected prosthetic implants with an emphasis on the organisms which form biofilms. J Clin Diagn Res: JCDR 7(2):219-223. https://doi.org/10.7860/JCDR/2013/4533.2732

8. Meinberg EG, Agel J, Roberts CS, Karam MD, Kellam JF (2018) Fracture and dislocation classification compendium-2018. J Orthop Trauma 32(Suppl 1):S1-S170. https://doi.org/10.1097/ BOT.0000000000001063

9. Charlson ME, Pompei P, Ales KL, MacKenzie CR (1987) A new method of classifying prognostic comorbidity in longitudinal studies: development and validation. J Chronic Dis 40(5):373-383. https://doi.org/10.1016/0021-9681(87)90171-8

10. Karczewski D, Winkler T, Renz N, Trampuz A, Lieb E, Perka C, Müller M (2019) A standardized interdisciplinary algorithm for the treatment of prosthetic joint infections. Bone Joint J 101B(2):132-139. https://doi.org/10.1302/0301-620X.101B2.BJJ2018-1056.R1

11. Izakovicova P, Borens O, Trampuz A (2019) Periprosthetic joint infection: current concepts and outlook. EFORT Open Rev 4(7):482-494. https://doi.org/10.1302/2058-5241.4.180092

12. Ochsner P, Borens O, Bodler P, Broger I, Eich G, Hefti F, Maurer T, Nötzli H, Seiler S, Suvà D (2016) Infections of the musculoskeletal system. In: Basic principles, prevention, diagnosis and treatment, 1st edn in English edn. Swiss orthopaedics and the Swiss Society for Infectious Diseases expert group "Infections of the musculoskeletal system", Grandvaux, Switzerland

13. Krenn V, Morawietz L, Perino G, Kienapfel H, Ascherl R, Hassenpflug GJ, Thomsen M, Thomas P, Huber M, Kendoff D, Baumhoer D, Krukemeyer MG, Natu S, Boettner F, Zustin J, Kölbel B, Rüther W, Kretzer JP, Tiemann A, Trampuz A, Gehrke T (2014) Revised histopathological consensus classification of joint implant related pathology. Pathol Res Pract 210(12):779-786. https://doi. org/10.1016/j.prp.2014.09.017

14. Diaz-Ledezma C, Higuera CA, Parvizi J (2013) Success after treatment of periprosthetic joint infection: a Delphi-based international multidisciplinary consensus. Clin Orthop Relat Res 471(7):2374-2382. https://doi.org/10.1007/s11999-013-2866-1
15. Zmistowski B, Tetreault MW, Alijanipour P, Chen AF, Della Valle CJ, Parvizi J (2013) Recurrent periprosthetic joint infection: persistent or new infection? J Arthroplasty 28(9):1486-1489. https:// doi.org/10.1016/j.arth.2013.02.021

16. Akgün D, Perka C, Trampuz A, Renz N (2018) Outcome of hip and knee periprosthetic joint infections caused by pathogens resistant to biofilm-active antibiotics: results from a prospective cohort study. Arch Orthop Trauma Surg 138(5):635-642. https:// doi.org/10.1007/s00402-018-2886-0

17. Berry DJ (1999) Epidemiology: hip and knee. Orthop Clin North Am 30(2):183-190. https://doi.org/10.1016/s0030-5898(05) 70073-0

18. Della Rocca GJ, Leung KS, Pape HC (2011) Periprosthetic fractures: epidemiology and future projections. J Orthop Trauma 25(Suppl 2):S66-S70. https://doi.org/10.1097/BOT.0b013e3182 $1 \mathrm{~b} 8 \mathrm{c} 28$

19. Beals RK, Tower SS (1996) Periprosthetic fractures of the femur: an analysis of 93 fractures. Clin Orthop Relat Res 327:238-246. https://doi.org/10.1097/00003086-199606000-00029

20. Lindahl H, Malchau H, Herberts P, Garellick G (2005) Periprosthetic femoral fractures classification and demographics of 1049 periprosthetic femoral fractures from the Swedish National Hip Arthroplasty Register. J Arthroplasty 20(7):857-865. https://doi. org/10.1016/j.arth.2005.02.001

21. Cook RE, Jenkins PJ, Walmsley PJ, Patton JT, Robinson CM (2008) Risk factors for periprosthetic fractures of the hip: a survivorship analysis. Clin Orthop Relat Res 466(7):1652-1656. https://doi.org/10.1007/s11999-008-0289-1

22. Janz V, Wassilew GI, Perka CF, Müller M (2018) Cerclages after femoral osteotomy are at risk for bacterial colonization during two-stage septic total hip arthroplasty revision. J Bone Joint Infect 3(3):138-142. https://doi.org/10.7150/jbji.24819

23. Guren E, Figved W, Frihagen F, Watne LO, Westberg M (2017) Prosthetic joint infection-a devastating complication of hemiarthroplasty for hip fracture. Acta Orthop 88(4):383-389. https:// doi.org/10.1080/17453674.2017.1301009

Publisher's Note Springer Nature remains neutral with regard to jurisdictional claims in published maps and institutional affiliations. 